\title{
A simple method for evaluating low-energy electron-molecule scattering cross sections using discrete basis functions $\dagger$
}

\author{
C W McCurdy Jrt, T N Rescigno and V McKoy \\ Arthur Amos Noyes Laboratory of Chemical Physics\|, California Institute of Tech- \\ nology, Pasadena, California 91125 , USA
}

Received 27 August 1975, in final form 10 October 1975

\begin{abstract}
We present a simple, approximate method for calculating low-energy electronmolecule scattering cross sections using only the results of a basis set diagonalization of the molecular Hamiltonian. The method is based on the approximate conservation of orbital angular momentum in collisions between slow electrons and molecules lacking a permanent dipole moment (low / spoiling). Results are presented for $\mathrm{e}^{-}-\mathrm{H}_{2}$ and $\mathrm{e}^{-}-\mathrm{N}_{2}$ in the static-exchange approximation.
\end{abstract}

\section{Introduction}

Electron-molecule scattering has long appeared to be a formidable problem due to the difficulties encountered in solving for continuum wavefunctions in the field of a non-spherical potential. Elastic electron scattering from a homonuclear diatomic molecule (the least anisotropic case) has been treated successfully at low energies by a variety of approaches of varying difficulty. For example, Tully and Berry (1969) numerically integrated the two-dimensional Schrödinger equation, Hara (1969) made use of prolate spheroidal coordinates, and several calculations have been made using single-centre expansions of the wavefunction (Temkin and Vasavada 1967, Lane and Henry 1969, Burke and Sinfailam 1970, Burke and Chandra 1972). Recently, more efficient methods have been developed which demonstrate that a knowledge of the wavefunction in the vicinity of the target is sufficient to calculate the scattering cross section (Rescigno et al 1975, Schneider 1975). In all these approaches, the coupling of orbital angular momentum by the non-spherical potential can be treated properly. However, a price of increased complexity over central-field calculations is inevitably paid for this capability.

The success of methods which employ purely square-integrable $\left(L^{2}\right)$ basis functions to treat rigorously scattering from non-spherical targets has led to even simpler,

\footnotetext{
† Supported by a grant from the National Science Foundation.

\$Present address: Department of Chemistry, University of California, Berkeley, California 94720, USA. $\$$ Present address: Lawrence Livermore Laboratory, University of California, Livermore, California 94550 , USA.

Contribution No 5161 .
} 
but less rigorous, attempts to describe electron-diatomic-molecule scattering. Among these are the Fredholm calculations of Winter and Lane (1975), and the method of Morrison and Lane (1975), both of which make use of the low $l$-spoiling approximation which we will discuss in the following section. In this paper we describe an exceedingly simple, but approximate, procedure for computing cross sections for low-energy elastic electron scattering from homonuclear diatomics. We present results for $\mathrm{e}^{-}-\mathrm{H}_{2}$ and $\mathrm{e}^{-}-\mathrm{N}_{2}$ scattering.

\section{Theory}

It is now well known that the square-integrable eigenfunctions at scattering energies obtained by diagonalizing the Hamiltonian for a spherical potential problem in a discrete basis are proportional to the true scattering solutions over the region spanned by the basis functions. Several approaches for resonant and non-resonant scattering make use of this fact, e.g. Hazi and Taylor (1970), Hazi and Fels (1971), Heller (1972). In general, it is necessary to develop a formula for the phaseshift which is independent of the normalization of the wavefunction. For example, the Harris formula (Harris 1967) can be viewed in this manner. These methods are unfortunately not rigorously applicable to scattering from a non-spherical potential. The coupling of various partial waves by the potential gives the problem of solving for the molecular scattering wavefunction in the body-fixed frame of the molecule, the same mathematical form as a multi-channel potential scattering problem. Thus at a given energy there is more than one regular solution to the radial equations and a linear combination of these must be taken to give the appropriate physical solutions. The diagonalization of the Hamiltonian in a discrete basis, however, gives only one solution at each eigenvalue. A possible method for circumventing this difficulty, requiring several diagonalizations of the Hamiltonian in different basis sets, has been discussed by Hazi (1973).

Alternatively, if we assume that orbital angular momentum is approximately conserved (low $l$ spoiling), then we will show how to extract scattering information from a single diagonalization of the Hamiltonian. Fano (1970) has discussed the basis for this approximation in the case of homonuclear diatomic molecules. The potential consists of the centrifugal barrier superposed on an attractive non-spherical potential of shorter range. It is known that the Rydberg series of molecules can be classified by the orbital angular momentum quantum number $l$ (Mulliken 1964); scattering solutions for electron-homonuclear-diatomic-molecule scattering have been similarly classified (Tully and Berry 1969, Temkin and Vasavada 1967). Fano (1970) notes that the approximate conservation of orbital angular momentum is not due to the nearly spherical symmetry of the molecular potential at large distances. A large asymmetry in the potential, even if it is of short range, is capable of coupling angular momenta. Instead, Fano (1970) concludes that the centrifugal barrier is probably responsible for preventing scattering solutions from penetrating the inner region of the potential and thus prevents the coupling of partial waves.

Approximate conservation of angular momentum should hold particularly well for homonuclear diatomics, because in those cases the centre of symmetry causes even values of $l$ to be uncoupled from odd values. Moreover, for linear molecules the projection of the angular momentum along the internuclear axis, $m_{l}$, is a good quantum number and leads to the uncoupling of the molecular symmetries $\Sigma, \Pi$, 
etc. On the other hand, one expects the low $l$-spoiling approximation to be less applicable to heteronuclear diatomics where $s$ and $p$ waves can couple and the centrifugal barrier may not be strong enough to keep them uncoupled.

We stress that the assumption of low $l$-spoiling does not imply that the scattering wavefunction can be thought of in terms of uncoupled partial waves at all $r$. The fact that in the exterior region the centrifugal barrier leads to uncoupling of the partial waves means that it will also confine some solutions to the interior region where coupling is appreciable. Indeed, the bound states of the potential show a great deal of anisotropy, and the requirement that the scattering solutions remain orthogonal to the bound states implies that there may be considerable anisotropy in the scattering solutions for small $r$. Consequently one expects that the partial-wave expansion of the molecular potential may converge very slowly.

In this paper we treat elastic electron scattering in the static-exchange approximation. Although Hara (1969) has shown that, in the case of $\mathrm{H}_{2}$, the inclusion of an adiabatic polarization potential substantially alters the cross section, we chose to neglect the effects of polarization of the target molecule to allow easy comparison of the present method with other work. We begin by diagonalizing the body-fixed Hartree-Fock Hamiltonian in a discrete basis. Within the framework of low $l$ spoiling, we may assume that the radial function, $R_{l m}(r)$, obtained by projecting with a spherical harmonic onto a positive energy $L^{2}$ eigenfunction, $\Psi(r)_{\text {molecular }}$,

$$
R_{l m}(r)=\int \mathrm{d} \Omega Y_{l m}^{*}(\Omega) \Psi(r)_{\text {moliculur }}
$$

is proportional to a scattering solution for $r$ less than some value, $b$, within the range of the basis set. With this assumption, a number of ways for extracting scattering information from $R_{l m}(r)$ suggest themselves.

One could use the value of $R_{l m}(r)$ at $r=b$ as the starting point for an outward numerical integration (Morrison and Lane 1975). If $b$ were sufficiently large, then only the direct, spherically symmetric part of the potential need be used in the integration. This procedure would be similar to the numerical $R$-matrix calculations of Burke et al (1971) and to the recent work of Morrison and Lane (1975). Alternatively, one could try fitting $R_{l m}(r)$ over the interval $[0, b]$ to another function that had the correct asymptotic form and extracting values of the tangent of the phaseshift in this manner. This procedure was first tried using a function of the form

$$
\chi(r)=\sum_{i} C_{i} \varphi_{i}(r)+A j_{l}(k r)+B n_{l}(k r)
$$

where $\varphi_{i}(r)$ is an $L^{2}$ function and $j_{l}$ and $n_{l}$ are spherical Bessel functions. This approach was found to be considerably less stable than the method finally adopted.

In the present work we chose to evaluate the phaseshift from the radial function and its derivative at a value of $r$. Consider the function

$$
\tan \delta_{l m}(r)=-\frac{R_{l m}(r) j_{l}^{\prime}(k r)-R_{l m}^{\prime}(r) j_{l}(k r)}{R_{l m}(r) n_{l}^{\prime}(k r)-R_{l m}^{\prime}(r) n_{l}(k r)} .
$$

If one were to plot this function for the exact (low $l$ spoiled) radial function, it would vary for small $r$, but then level off to a constant value as the asymptotic region were approached. This should also be the case for a basis set representation of $R_{l m}(r)$, provided the basis spans a sufficiently broad region of configuration space. Then $\tan \delta_{l m}(r)$ should approach some stable value and maintain this value until 
the basis set was no longer capable of representing $R_{l m}(r)$ accurately. Formulae for the cross section in terms of the low $l$-spoiled phaseshifts can be found in Temkin and Vasavada (1967).

\section{Computational}

We begin by constructing the Hartree-Fock wavefunction for the target molecule, which defines the static-exchange potential for the scattering calculation. This part of the procedure simply involves performing a standard basis set self-consistent field (SCF) calculation to obtain the occupied orbitals of the molecular target. The bodyfixed static-exchange Hamiltonian (for closed-shell targets) is then given by

$$
H^{\mathrm{HF}}=-\frac{1}{2} \nabla^{2}+V^{\text {direct }}+V^{\text {exchange }}
$$

where

$$
V^{\text {direct }}(\boldsymbol{r})=-\sum_{\text {nuclei }} \frac{Z_{n}}{\left|\boldsymbol{r}-\boldsymbol{R}_{n}\right|}+2 \sum_{\alpha} \int \frac{\mathrm{d} \boldsymbol{r}^{\prime} \phi_{\alpha}^{*}\left(\boldsymbol{r}^{\prime}\right) \phi_{\alpha}\left(\boldsymbol{r}^{\prime}\right)}{\left|\boldsymbol{r}-\boldsymbol{r}^{\prime}\right|}
$$

and

$$
V^{\mathrm{ex}}\left(\boldsymbol{r}, \boldsymbol{r}^{\prime}\right) F\left(\boldsymbol{r}^{\prime}\right)=-\sum_{\alpha} \int \frac{\mathrm{d} \boldsymbol{r}^{\prime} \phi_{\alpha}^{*}\left(\boldsymbol{r}^{\prime}\right) \phi_{\alpha}(\boldsymbol{r}) F\left(r^{\prime}\right)}{\left|\boldsymbol{r}-\boldsymbol{r}^{\prime}\right|}
$$

where $\phi_{\alpha}$ is an occupied space orbital.

The validity of the low $l$-spoiling approximation implies that we should be able to construct eigenfunctions of $H^{\mathrm{HF}}$ which for large $r$ are proportional to a single $Y_{l m}$. To this end we diagonalize $H^{\mathrm{HF}}$ with a different basis set of Gaussian functions for each value of $l$ and $|m|$ under consideration. This basis is chosen by augmenting the basis used to perform the SCF calculation with additional diffuse functions of one particular symmetry most of which are placed at the centre of the molecule. Such a choice guarantees that the positive energy eigenfunctions of $H^{\mathrm{HF}}$ will be orthogonal to an accurate set of non-spherical occupied orbitals and will also go asymptotically as a particular $Y_{l m}$. We emphasize that the SCF equations need be solved only once to define $H^{\mathrm{HF}}$ and that this Hamiltonian is then rediagonalized for each symmetry desired.

The choice of Gaussian basis functions makes the evaluation of the projection integral of equation (1) particularly simple. In a coordinate system in which the $Z$ axis is chosen to be the internuclear axis, the projection of a spherical harmonic at the midpoint of the molecule onto a Gaussian located on the internuclear axis at a distance $A$ from the origin can be written in the form,

$$
\begin{aligned}
\int Y_{l m}^{*}(\theta, \varphi) x^{i} y^{j}(z-A)^{k} \exp \left[-\alpha(r-A)^{2}\right] \mathrm{d} \Omega \\
=\exp \left[-\alpha\left(r^{2}+A^{2}\right)\right] \int Y_{l m}^{*}(\theta, \varphi)(r \sin \theta \cos \varphi)^{i}(r \sin \theta \sin \varphi)^{j}(r \cos \theta-A)^{k} \\
\quad \times \exp (2 \alpha r A \cos \theta) \sin \theta \mathrm{d} \theta \mathrm{d} \varphi .
\end{aligned}
$$

This integral was evaluated analytically for the various cases we considered. Phaseshifts were then extracted using equation (3) as described previously. 


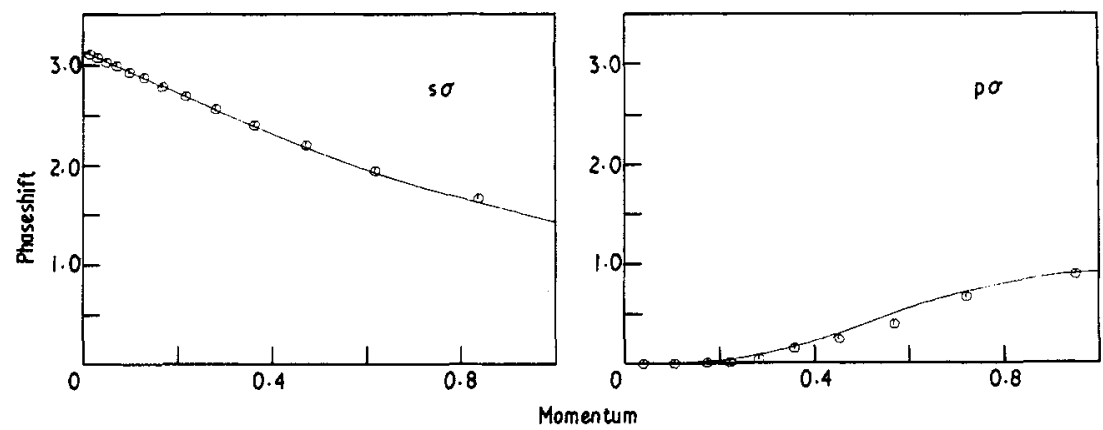

Figure 1. Comparison of static-exchange phaseshifts from equation (3) (circles) for $\mathrm{e}^{-}-\mathrm{H}_{2}$ scattering with the low $l$-spoiling results of Schneider (1975). (Momentum in atomic units.)

\section{Results}

We computed s and $\mathrm{p} \sigma$ phaseshifts for molecular hydrogen as a test of the accuracy of the method. The basis used was a set of 22 s-type Gaussians on each hydrogen, which was used in a previous calculation (Rescigno et al 1975). The results are compared with the $R$-matrix results of Schneider (1975), which were also computed in the $l$-spoiling approximation, in figure 1.

The Hartree-Fock occupied orbitals for $\mathrm{N}_{2}$ were computed using a basis of [4s 3p] Gaussians contracted from a $(9 s 5 p)$ set of primitives suggested by Dunning (1970). This basis was augmented by two $\mathrm{d} \pi$ and $\mathrm{d} \sigma$ polarization functions (Truhlar et al 1972). The polarization functions are essential to the proper description of the quadrupolar part of the molecular potential (Truhlar et al 1972).

To calculate the $\Sigma_{\mathrm{g}}$ virtual orbitals from which s-wave phaseshifts were computed, this basis was augmented by three additional s-type Gaussians on each nitrogen and seven diffuse s-type Gaussians at the midpoint with exponents chosen in a geometric series. The $\Sigma_{u}$ basis consisted of these same s-type functions on the nitrogens used for $\Sigma_{\mathrm{g}}$, and in addition, five uncontracted $z$-type Gaussians were placed on each nitrogen, seven diffuse $z$ Gaussians at the midpoint, and two $\mathrm{d}_{z 2}$ Gaussians on the nitrogens. For $\Pi_{\mathrm{u}}$ we used seven primitive $x$-type Gaussians on each nitrogen with

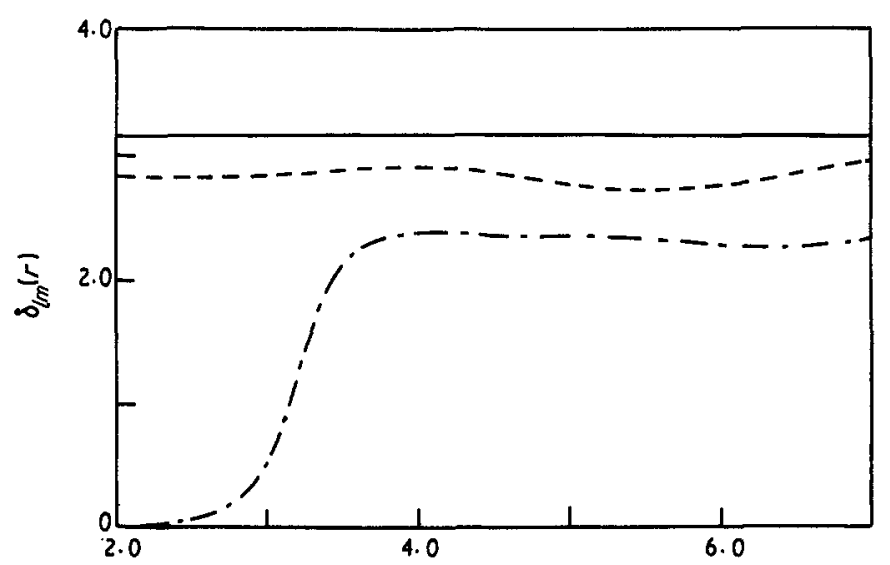

Figure 2. Some representative plots of $\delta_{l m}(r)$ from equation (3). The full, broken and chain curves are for orbitals of $s \sigma, p \pi$ and $d \pi$ symmetry respectively. 
Table 1. $\mathrm{e}^{-}-\mathrm{N}_{2}$ phaseshifts.

\begin{tabular}{|c|c|c|c|c|c|c|c|}
\hline \multicolumn{2}{|c|}{$\mathrm{s}$} & \multicolumn{2}{|c|}{$\mathrm{p} \sigma$} & \multicolumn{2}{|c|}{$\mathrm{p} \pi$} & \multicolumn{2}{|c|}{$\mathrm{d} \pi$} \\
\hline$k(\mathrm{au})$ & $\delta_{00}$ & $k(\mathrm{au})$ & $\delta_{10}$ & $k(\mathrm{au})$ & $\delta_{11}$ & $k(\mathrm{au})$ & $\delta_{21}$ \\
\hline 0.017 & $3 \cdot 101$ & 0.0403 & $3 \cdot 141$ & 0.039 & $3 \cdot 141$ & 0.047 & $0 \cdot 0$ \\
\hline 0.037 & 3.05 & 0.079 & $3 \cdot 139$ & 0.072 & $3 \cdot 140$ & 0.082 & 0.0 \\
\hline 0.066 & $2 \cdot 98$ & 0.135 & $3 \cdot 127$ & 0.114 & $3 \cdot 136$ & 0.127 & 0.0 \\
\hline 0.109 & $2 \cdot 88$ & 0.222 & 3.09 & 0.172 & $3 \cdot 12$ & 0.187 & 0.0 \\
\hline $0 \cdot 179$ & $2 \cdot 72$ & 0.361 & $2 \cdot 95$ & $0 \cdot 253$ & 3.09 & 0.273 & 0.01 \\
\hline $0 \cdot 296$ & 2.45 & 0.577 & 2.66 & 0.372 & $3 \cdot 01$ & 0.393 & 0.06 \\
\hline 0.491 & $2 \cdot 09$ & 0.861 & $2 \cdot 19$ & 0.553 & $2 \cdot 83$ & 0.518 & 0.90 \\
\hline \multirow[t]{3}{*}{0.815} & 1.60 & 1.098 & $1 \cdot 7$ & 0.830 & 2.55 & 0.631 & $2 \cdot 35$ \\
\hline & & & & & & 0.894 & $2 \cdot 52$ \\
\hline & & & & & & $1 \cdot 182$ & $2 \cdot 33$ \\
\hline
\end{tabular}

eight more at the midpoint and two $\mathrm{d} \pi$ Gaussians on the nitrogens. The $\Pi_{\mathrm{g}}$ basis consisted of seven $x$-type Gaussians on each nitrogen, twelve $x z$-type Gaussians at the midpoint, and two $\mathrm{d} \pi$ functions on the nitrogens. From these four diagonalizations of the Hartree-Fock Hamiltonian we can extract the $\mathrm{s}, \mathrm{p} \sigma, \mathrm{p} \pi$ and $\mathrm{d} \pi$ phaseshifts. In figure 2 we show some representative plots of $\delta_{l m}$ obtained from equation (3) as a function of $r$. These demonstrate the occurrence of a region in which the phaseshift is constant.

Table 1 lists the s, $\sigma, \mathrm{p} \pi$ and $\mathrm{d} \pi$ phaseshifts. In figures 3 and 4 we compare our results with those of Burke and Sinfailam (1970). We plot both the arctangent of the appropriate element of the reactance matrix from Burke and Sinfailam (1970), as well as their largest eigenphase. Note that in the complete absence of angular momentum coupling these two quantities would be identical. The extent to which they coincide is an indication of the degree of accuracy of the low $l$-spoiling approximation. There is reasonably good agreement between the results we find here and those of Burke and Sinfailam (1970). It is not clear whether the discrepancies between the two calculations are due to the approximate nature of the method we employed or to the fact that Burke and Sinfailam (1970) were forced to retain only a small number of terms in their one-centre expansion of the potential.

A more recent calculation on $\mathrm{e}^{-}-\mathrm{N}_{2}$ by Burke and Chandra (1972) shows that the $\Sigma_{g}$ eigenphase increases as the number of terms in a single-centre expansion

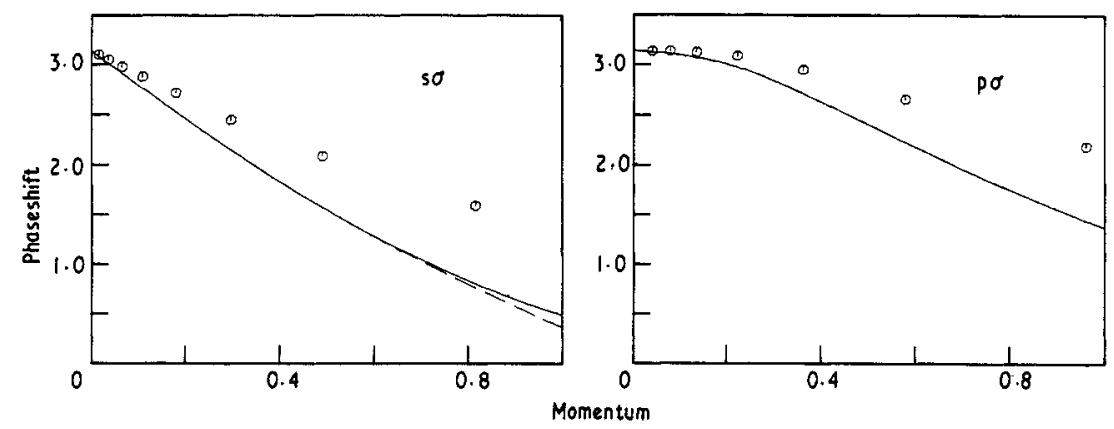

Figure 3. Comparison of the $\mathrm{s} \sigma$ and $\mathrm{p} \sigma$ phaseshifts of this work (circles) for $\mathrm{e}^{-}-\mathrm{N}_{2}$ scattering with the largest eigenphase (full curve) and arctangent of the appropriate diagonal element of the reactance matrix (broken curve) of Burke and Sinfailam (1970). The curves for $p \sigma$ are indistinguishable on this scale. (Momentum in atomic units.) 


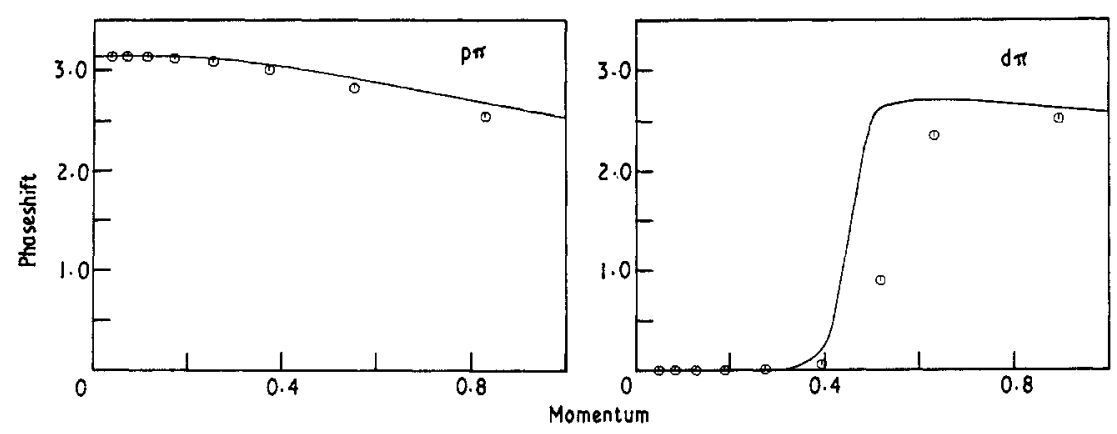

Figure 4. Results for $\mathrm{p} \pi$ and $\mathrm{d} \pi$ phaseshifts (circles) and the results of Burke and Sinfailam (1970) (full curve) for $\mathrm{N}_{2}$. On this scale the largest eigenphase and the arctangent of the diagonal reactance matrix element are indistinguishable. (Momentum in atomic units.)

of the potential increases. However, these calculations were performed by dropping the exchange parts of the potential and replacing them with Lagrange multipliers to enforce orthogonality to the occupied orbitals. Nevertheless, we might expect that the $\Sigma_{\mathrm{g}}$ phaseshifts of Burke and Sinfailam (1970) would show the same behaviour as the number of terms in the potential expansion was increased, bringing their results into closer agreement with ours. Unfortunately, Burke and Chandra (1970) do not show the dependence of the $\Sigma_{\mathrm{u}}$ and $\Pi_{\mathrm{u}}$ eigenphases on the number of terms in the expansion of the potential.

Some comments are in order concerning the resonance in the $\Pi_{\mathrm{g}}-\mathrm{d} \pi$ phaseshift. Burke and Chandra (1972) found it necessary to include a polarization potential to produce a sharp resonance in the $\Pi_{\mathrm{g}}$ wave. We find a $\Pi_{\mathrm{g}}$ resonance in the present calculation and expect that the neglect of exchange effects for this symmetry in the calculation of Burke and Chandra (1972) was responsible for its disappearance as a shape resonance. Our calculations show a resonance in the $\mathrm{d} \pi$ phaseshift at $3.54 \mathrm{eV}$ (a momentum of $0.51 \mathrm{au}$ ), compared to Burke and Sinfailam's (1970) value of $2.80 \mathrm{eV}$ (a momentum of $0.454 \mathrm{au}$ ). We conclude that the low-energy $\Pi_{\mathrm{g}}$ resonance is a shape resonance which can be described by the static-exchange model, but that the position of the resonance is affected by correlation to the extent that the static-exchange resonance is about $1 \mathrm{eV}$ too high (Golden 1966).

\section{Summary and conclusions}

We have described a simple method for calculating low-energy elastic electron-molecule cross sections. The method depends on the validity of the low $l$-spoiling approximation and the ability of a discrete basis set to describe a molecular continuum wavefunction over a sufficiently large region of space. The calculations reported here were limited to the static-exchange approximation, but this restriction can be relaxed. The method should be applicable to elastic electron scattering from any homonuclear diatomic molecule as well as from small molecules with no permanent dipole moment.

\section{References}

Burke P G and Chandra N 1972 J. Phys. B: Atom. Molec. Phys. 5 1696-711

Burke P G, Hibbert A and Robb W D 1971 J. Phys. B: Atom. Molec. Phys. 4 153-61 
Burke P G and Sinfailam A L 1970 J. Phys. B: Atom. Molec. Phys. 3 641-59

Dunning T H 1970 J. Chem. Phys. 53 2823-33

Fano U 1970 Comments on Atomic and Molecular Physics vol 1 (New York: Academic Press) pp 140-4 Golden D E 1966 Phys. Rev. Lett. $17847-8$

Hara S 1969 J. Phys. Soc. Japan 196927 1009-19

Harris F E 1967 Phys. Rev. Lett. $19173-5$

Hazi A U 1973 Chem. Phys. Lett. 20 251-6

Hazi A U and Fels M F 1971 Chem. Phys. Lett. 8 582-6

Hazi A U and Taylor H S 1970 Phys. Rev. A 1 1109-20

Heller E J 1972 Phys. Rev. A 6 1247-8

Lane N F and Henry R J W 1969 Phys. Rev. 183 221-31

Morrison M A and Lane N F 1975 Phys. Rev. A to be published

Mulliken R S $1964 \mathrm{~J}$. Am. Chem. Soc. 86 3183-97

Rescigno T N, McCurdy C W and McKoy V 1975 Phys. Rev. A 11 825-9

Schneider B 1975 Phys. Rev. A 11 1957-62

Temkin A and Vasavada K V 1967 Phys. Rev. 160 109-17

Truhlar D G, Van-Catledge F A and Dunning J H 1972 J. Chem. Phys, 57 4788-99

Tully J C and Berry R S 1969 J. Chem. Phys. 51 2056-75

Winter T G and Lane N F 1975 Chem. Phys. Lett. $30363-6$ 